\title{
Establishment of a finite element model of supination-external rotation ankle joint injury and analysis of mechanical changes in the posterior malleolar surface
}

\section{Xin Zhang}

Jiading District Central Hospital Affiliated Shanghai University of Medicine \&Health Sciences

\section{Pinliang Xie}

Jiading District Central Hospital Affiliated Shanghai University of Medicine \&Health Sciences

\section{Weirong Shao}

Jiading District Central Hospital Affiliated Shanghai University of Medicine \&Health Sciences

\section{Ming Xu}

Jiading District Central Hospital Affiliated Shanghai University of Medicine \&Health Sciences

\section{Xiaoping Xu}

Jiading District Central Hospital Affiliated Shanghai University of Medicine \&Health Sciences

Yong Yin ( $\nabla$ yinyong910428@126.com )

Jiading District Central Hospital Affiliated Shanghai University of Medicine \&Health Sciences

\section{Lan Fei}

Jiading District Central Hospital Affiliated Shanghai University of Medicine \&Health Sciences

\section{Article}

Keywords: Supination-external rotation, Stress distribution, Finite element analysis, Posterior malleolus

Posted Date: May 31st, 2022

DOI: https://doi.org/10.21203/rs.3.rs-1036053/v2

License: (c) (i) This work is licensed under a Creative Commons Attribution 4.0 International License. Read Full License 


\section{Abstract}

Background By establishing a three-dimensional finite element model of supination and external rotation ankle injury, the stress characteristics of the posterior ankle joint surface can be obtained, and complete analysis of the corresponding stress on the lateral ankle can be conducted.

Methods Thin-layer computed tomography (CT) images of normal ankle joints in the supination and external rotation nonweight-bearing states were selected, a three-dimensional data model of each ankle joint, including the ligament, was established, and whether different stages of injury coexisted with lateral ankle fracture was analysed by the finite element method. A load was applied to examine different ankle joint stress values and pressure distributions on the surface of the posterior ankle joint.

Results When a load was applied, the maximum stress was located at the point of attachment of the anterior tibiofibular ligament to the tibia. When the anterior tibiofibular ligament was removed and the lateral malleolus was intact, the maximum stress (271.2 MPa) was located at the attachment point of the posterior tibiofibular ligament to the tibia, and the maximum pressure of the posterior ankle joint surface was $2.626 \mathrm{MPa}$. When a lateral malleolar fracture was present and the same load was applied, the maximum stress ( $82 \mathrm{MPa}$ ) was located on the fibular fracture surface, and the maximum pressure of the posterior ankle joint surface was $7.787 \mathrm{MPa}$. The posterior tibiofibular ligament was then removed completely from the lateral malleolus, upon which the maximum stress (132.7 MPa) was located at the point of attachment of the posterior tibiofibular ligament to the fibula and the maximum pressure of the posterior ankle joint surface was $4.505 \mathrm{MPa}$. When a lateral malleolar fracture was present, the maximum stress (82.72 MPa) was located on the fibular fracture surface, and the maximum pressure of the posterior ankle joint surface was $8.022 \mathrm{MPa}$.

Conclusion This study shows that reconstruction of the lateral malleolus in supination-external rotation ankle injury significantly affects the stress distribution at the posterior malleolar joint surface. When reconstruction of the lateral malleolus is complete, the pressure distribution of the posterior malleolar joint surface can be significantly reduced. The disappearance of pressure concentration helps reduce the stresses associated with posterior ankle fracture. At this time, the rotational stress imposed by posterior ankle fracture is significantly higher than the vertical stress; the former should be the focus of clinician attention to ensure optimal healing.

\section{Background}

Ankle fractures account for approximately $3.9 \%$ of all systemic fractures and are a common type of intraarticular fracture. Approximately $14-44 \%$ of these fractures impact the posterior malleolus, and they often result in ankle instability ${ }^{1}$. The posterior malleolus refers to the structures behind the fibular notch of the distal tibia, posterior tubercle (Volkmann tubercle), ankle sulcus and posterior colliculus of the medial malleolus. It is an integral part of the distal tibiofibular complex, increasing the contact area of the tibiotalar joint, reducing the pressure per unit area of the tibiotalar joint, preventing backwards movement 
of the talus, and supporting and maintaining the stability of the ankle joint ${ }^{2,3}$. Most posterior ankle fractures are associated with lateral ankle fractures and ligament injuries around the ankle, especially supination-external rotation ankle fractures caused by severe rotation ${ }^{4}$. It is rare that they appear alone; if the reduction is not good, the area is prone to traumatic arthritis, affecting the function of the ankle joint ${ }^{5,6}$.

In the treatment of supination-external rotation ankle joint injury, anatomical reconstruction and rigid fixation of lateral ankle fractures are usually not difficult to carry out, but controversy exists regarding the type and effectiveness of fixation for posterior ankle fracture ${ }^{7}$. Current opinion holds that some posterior malleolar blocks, although small, have an important role in maintaining the stability of the lower tibiofibular ${ }^{8}$. Thus, exploring how the mechanical changes before and after external ankle reconstruction for the posterior ankle facet directly affect the stability of the posterior ankle fracture fragment and indirectly affect the lower tibiofibular and overall stability of the ankle is essential. The final results of such an analysis may suggest new ideas for the treatment of posterior ankle fractures in supinationexternal rotation-type injuries.

In this model, a reverse three-dimensional model is constructed through the foot supination posture, and the setting of this posture is consistent with the foot posture of supination and external rotation injury. We used rupture of the anterior tibiofibular ligament to simulate injury of stage I of supination and external rotation; we simulated injury of stage II of supination and external rotation by constructing the fibular fracture line from the back up to the front down; and we simulated injury of stage III of supination and external rotation through rupture of posterior tibiofibular ligament. In addition, a complete injury model of the lateral malleolus was set up for the stage III injury. After applying pressure, we explored and analysed the stress changes of the posterior ankle joint surface.

Based on this study, we can determine how the integrity of the lateral malleolus affects the stability of the posterior malleolus and what kind of instability. This will help clinicians understand the mechanical changes after lateral malleolus reconstruction, remind them to pay attention to the rotational instability of the posterior malleolus and solve existing clinical problems. It provides a biomechanical basis for the fixation of the posterior malleolus after the reconstruction of the lateral malleolus.

\section{Materials And Methods}

\section{Acquisition of ankle CT images}

One healthy adult volunteer with no previous history of injury, such as ankle fracture or dislocation, or pathological conditions, such as ankle arthritis, bone disease or bone tumour, was selected. The right ankle joint of the volunteer ( $60 \mathrm{~kg}$ and 28 years old) was imaged with thin-section CT, with a scanning layer thickness of $0.625 \mathrm{~mm}$, resulting in 657 images of $512 \times 512$ pixels that were saved in DICOM format. During the CT scan, the volunteer's ankle was nonweight-bearing in the supination position.

\section{$23 \mathrm{D}$ reconstruction and optimization}


DICOM format images were imported into Mimics 21.0 software (Materialise, Belgium); the images were segmented; and the tibia, talus, and fibula were reconstructed. Multiple bones (including the calcaneus, navicular cuneus, mediolateral cuneus, dice cuneus, and mediolateral cuneus) beneath the talus were fused to reconstruct their 3D models (Fig. 1). The following processes were carried out in proper sequence: (a) Automatic threshold segmentation and differentiation are carried out according to the grey value of different tissues, and the bone tissue is preliminarily separated, (b) a mask is used to establish the structure model of each part, (c) the manual layer editing tool is used to eliminate the redundant part or fill in the missing part, and (d) the model is wrapped and smoothed, the hole and smooth surface are filled, the corresponding three-dimensional model is preliminarily established, and the model data file is exported in STL format.

We import the STL format file generated by Mimics into Geomagic Studio 2017 software, erase the nails and redundant features of the model, and then smooth the model. Then, the accurate surface module is used to detect the contour line of the model, any deformed or unreasonable contour line is edited, and additional contour line is added appropriately to facilitate the generation of surface patches. After the surface patches are generated successfully, the surface is fitted, and the fitted model is exported to a general STEP format model data file.

\section{Establishment of the finite element model}

We import the optimized 3D models into SolidWorks software, carry out feature recognition and surface diagnosis on the geometric model, repair the problematic surface, construct the articular cartilage model by using the stretching and segmentation commands on the part interface, draw 3D lines in the 3D sketch to simulate the ligament model, and finally establish a complete three-dimensional ankle model including tibia, fibula, talus, calcaneus, cartilage and ligament and then save the model as a 3D geometry file in X_ T format. The obtained geometric models are imported into ABAQUS software (2018, Dassault, Providence, $\mathrm{RI}, \mathrm{USA}$ ) to build finite element models with the help of the attachment points and anatomical locations of ligaments determined from reference documentation; there were a total of 362,351 nodes and 261,420 units (Fig. 1). Bone and ligaments are simplified into isotropic, homogeneous linear elastic materials, and the material parameters are listed in Table $1^{9,10}$. In brief, bonding contact is used between the ligament and bone, calcaneus and talus, while face-to-face contact is used for the talus, tibiofibula and calcaneus cartilage and bone. The friction formula is the penalty function algorithm, the normal contact stiffness is "hard" contact, and the friction coefficient is 0.2 to establish the finite element analysis model.

\section{Research on grid convergence}

The normal supination and external rotation ankle joint model is divided into grids of different levels. The grid levels are divided into coarse, semi coarse, fine and very fine. Then, the same boundary conditions and loads are applied to the four grid models. The stress and displacement results obtained from the analysis are shown in the table below (Table 2): 
Table 1

Material parameters

\begin{tabular}{|lll|}
\hline & Elastic modulus (MPa) & Poisson's ratio \\
\hline Bone & 14000 & 0.3 \\
\hline Cartilage & 15 & 0.46 \\
Ligament & 260 & 0.49 \\
\hline
\end{tabular}

Table 2

Research on grid convergence

\begin{tabular}{|lll|}
\hline Grid level & Element quantity & Max. stress (MPa) \\
\hline coarse & 143507 & 68.5 \\
\hline semi coarse & 201317 & 95.55 \\
fine & 264023 & 83.73 \\
very fine & 369803 & 77.76 \\
\hline
\end{tabular}

It can be seen from the above table that the stress results of the fine grid level are similar to those of the very fine grid level, so the subsequent analysis adopts the fine grid model as the finite element analysis model based on the comprehensive consideration of calculation accuracy and calculation time.

\section{Verification and analysis of the finite element model}

In this part, the model loading parameters are based on a fixed lower surface of the talus, and a dead weight loaded between the tibia and proximal fibula and the internal rotation force are used to simulate the post rotation-external rotation-type injury situation. Three directional fixation restraints are set with full degrees of freedom in XYZ at the under-surface of the talus, and a reference point is established near the upper surface of the tibia and fibula, coupled with the upper surface degrees of freedom, the application of a dead weight load (480 N compression on the upper surface of the tibia and $120 \mathrm{~N}$ compression on the upper surface of the fibula) and an internal rotation force (gradually increasing internal rotation force, simulating an IV stage injury). A $600 \mathrm{~N}$ vertical compressive load is applied to the upper sections of the lower tibia and fibula of the model, where the calcaneus is fixed and the talus is constrained (Fig. 2). The maximum contact stress of the ankle joint surface is $2.1059 \mathrm{MPa}$, the contact area is $373.658 \mathrm{~mm}^{2}$ (Fig. 3), and the model is validated as effective ${ }^{11}$. The calculations of outcomes are based on the maximum stress location and pressure on the articular surface.

\section{Results}




\section{Model of stage I supination-external rotation ankle injury}

The maximum value of stress at $1.5 \mathrm{~N} \cdot \mathrm{m}$ internal rotation was $51.05 \mathrm{MPa}$ at the attachment point of the anterior tibiofibular ligament to the fibula, and the maximum value of pressure at the posterior ankle joint surface was $2.549 \mathrm{MPa}$ (Fig. 4). This is consistent with the description of Lauge-Hansen typing in the clinic $^{12,13}$.

By removing the anterior tibiofibular ligament, the lateral malleolus remained intact. At this time, we continued to load a $10 \mathrm{~N} \cdot \mathrm{m}$ internal rotation force. The calculation results showed that when loading this force, the maximum stress was located at the tibial attachment point of the posterior tibiofibular ligament, which was $271.2 \mathrm{MPa}$, and the maximum pressure of the posterior ankle joint surface was 2.626 MPa (Fig. 5).

\section{Model of stage II supination-external rotation ankle injury}

The fibula fracture line was drawn posterosuperior and inferior to construct the stage II injury fracture model (Fig. 6). The calculation showed that the maximum stress on the fibula fracture surface when a 10 $\mathrm{N} \cdot \mathrm{m}$ internal rotation force was applied was $82 \mathrm{MPa}$; the maximum pressure at the posterior ankle joint surface was 7.787 MPa (Fig. 7).

Damage to the supination and external rotation developed from stage I to stage II. Under the same load, the maximum stress changed from the tibial attachment point of the posterior tibiofibular ligament to the fracture end of the lateral malleolus. The maximum pressure of the posterior ankle joint surface increased from 2.626 MPa to $7787 \mathrm{MPa}$. The location of highest stress of the posterior malleolus moved back significantly.

\section{Model of stage III supination-external rotation ankle injury}

With the lateral malleolus intact, a model of stage III injury was established by removing the posterior tibiofibular ligament. Loading with internal rotation was continued, and the calculated results indicated that the maximum stress after removing the posterior tibiofibular ligament was $132.7 \mathrm{MPa}$, located at the attachment point of the posterior talofibular ligament to the fibula, and the maximum pressure on the posterior ankle joint surface was 4.505 MPa (Fig. 8).

On the basis of the above, fibula fracture lines were drawn from upwardss and forward from the back to construct a fracture model. The calculation results showed that the maximum stress was $82.72 \mathrm{MPa}$, located at the fibula fracture surface, and the maximum value of pressure at the posterior ankle joint surface was $8.022 \mathrm{MPa}$ (Fig. 9).

The damage of supination and external rotation developed from stage II to stage III. Under the same load, the positions of the maximum stress did not change, which were all fibular fracture surfaces. The maximum pressure of the posterior ankle joint surface increased from $7.787 \mathrm{MPa}$ to $8.022 \mathrm{MPa}$. The maximum stress position of the posterior malleolus did not change significantly. 
In the case of stage III injury with an intact lateral malleolus, under the same load, the maximum stress was located at the fibular attachment point of the posterior peroneal ligament. The maximum pressure of the posterior ankle joint surface decreased significantly to $4.505 \mathrm{MPa}$. There was no obvious stress concentration in the stressed part of the posterior malleolus.

\section{Discussion}

Lauge-Hansen's classification, which was published in a 1950 issue of Archives of Surgery, has become one of the most widely used ankle fracture classification systems. In that article, Niel Lauge-Hansen presented an ankle fracture classification and an explanation for low-energy fractures caused by severe rotation of the ankle ${ }^{13}$. This classification is based on foot position at the time of the traumatic event (supination or pronation) and the direction of the deforming forces (abduction, adduction, or external rotation). Seventy percent of these fractures were of the supination-external rotation type: the foot was in the supination position due to external severe rotation. Stage III-IV damage involved bony structures or associated ligaments of the posterior malleolus ${ }^{14}$.

Through three-dimensional heat map analysis, Yu Tao et al. found that most of the fracture lines of the posterior malleolus were concentrated in an arc-banded region that started from $1 / 7$ to $2 / 7$ of the tangent line of the posterior edge and ended at $5 / 11$ to $7 / 11$ of the tangent line of the outer edge ${ }^{15}$. The proportion of posterior malleolar fracture blocks to the total articular surface of the distal tibia was $14.96 \%[10]$. In the past, a posterior malleolar fracture area of more than $25 \%$ was considered to be an indication for surgical treatment of posterior malleolar fracture. Verhage et al. found that the incidence of osteoarthritis was approximately $48 \%$ when the area of posterior malleolar fracture accounted for $5 \%-25 \%$ of the tibial articular surface and as high as $54 \%$ when the area was greater than $25 \%$, and excellent reduction could not be maintained except by internal fixation ${ }^{5,16}$.

Mangnus et al. concluded that the position of the posterior malleolar fracture line may have a greater impact on stability than the size of the fracture area; even if the posterior malleolar fracture is small, it affects ankle joint stability ${ }^{17}$. Gardner et al. confirmed on postoperative CT that posterior malleolar fracture affects the stability of the inferior tibiofibular ligament and that unfixed posterior malleolar fracture can lead to tibiofibular joint subluxation ${ }^{18,19}$. In recent years, some studies have found that fixation of posterior ankle fracture blocks can restore the tension of the lower tibiofibular posterior ligament and improve the reduction of lower tibiofibular syndesmosis ${ }^{20}$. The stability obtained is better than that of lower tibiofibular screw fixation. Therefore, some scholars have even reported that as long as there is a posterior ankle fracture, no matter how large the fracture block is, anatomical reconstruction should be carried out to reduce the use of lower tibiofibular screws ${ }^{21}$. Therefore, for the treatment of posterior malleolar fractures, determining whether surgery is necessary by the size of the bone only is not reliable.

Based on the data in our experiment, it can be concluded that anatomical reconstruction and rigid fixation of the lateral malleolus can significantly reduce the stress of the posterior malleolus in supination and 
external rotation ankle injury. Furthermore, the completion of anatomical reconstruction moves the maximum distribution of stress change from the broken end of the fracture to the ligament attachment point, and the prominent concentration of the maximum stress part of the posterior ankle clearly disappears. This means that the integrity of the lateral malleolus in supination external rotation ankle injury significantly reduces the vertical instability of the posterior malleolus under the action of stress, and the rotational instability caused by the traction of the ligament becomes more prominent, with a maximum stress ratio of 132.7:4.5. The disappearance of obvious stress concentrations in the posterior malleolus is consistent with the phenomenon of self-reduction of posterior malleolar fractures after lateral malleolar reduction and reconstruction. Therefore, for posterior ankle fractures, the establishment of rotational stability may be more important than vertical stability. If the stability of posterior ankle fracture and posterior tibiofibular ligament is not satisfactory, the maximum contact stress of the posterior ankle joint surface will increase to more than twice that of normal people, which will further aggravate the instability of the ankle joint. Therefore, in supination external rotation ankle injury, the integrity of the lateral malleolus has an important impact on the stability of the posterior structure of the ankle, which coincides with the views of mangnus, Gardner and others ${ }^{17,18}$. Regarding the choice of internal fixation method for posterior ankle fracture, two screws and gaskets have good anti rotation capacity, while buttress plates have better anti vertical capacity. Therefore, the selection of two screws and gaskets for internal fixation may be sufficient for posterior ankle fracture in supination and external rotation ankle injury after lateral ankle reconstruction, but this needs to be proven by further biomechanical research.

\section{Conclusion}

In conclusion, this study shows that the external ankle integrity of supination external rotation ankle injury is of great significance to the stress change of the posterior ankle joint surface. When the lateral malleolus is intact, the pressure distribution on the posterior ankle joint surface can be significantly reduced, and the disappearance of pressure concentration is helpful to reduce the stress associated with posterior ankle fracture. At this time, the rotational stress experienced by posterior ankle fracture is significantly higher than the vertical stress, and clinicians should pay more attention to the former in treatment. A deficiency of this study is that the simplified simulation model differs from the actual situation. The ligament is not a linear elastic material, and its displacement load curve presents multiphase characteristics. In different pretension states, its stiffness is different. In the state of supination lateral preload, the material parameters of the external collateral ligament may be different from those of other ligaments in the simplified simulation model of finite element simulation. Moreover, no further research has been conducted on $\mathrm{IV}^{\circ}$ injuries involving triangular ligament and medial malleolar fractures, the construction of posterior malleolar fractures and the selection of internal fixation.

\section{Declarations}

Ethics approval and consent to participate 
This study was conducted in keeping with the Declaration of Helsinki and has been reviewed and approved by the ethics committee of Jiading District Central Hospital Affiliated Shanghai University of Medicine \&Health Sciences, all methods were carried out in accordance with relevant guidelines and regulations. Written informed consent was obtained from the healthy adult prior to enrollment in the study as per our study protocol reviewed and approval by local institutional review boards.

\section{Consent for publication}

All subjects were informed and agreed to publish identification information/images in online open-access publications.

\section{Availability of data and materials}

The datasets generated and/or analysed during the current study are not publicly available due to patient personal health confidentiality agreement but are available from the corresponding author on reasonable request.

\section{Competing interests}

The authors declared that they have no conflict of interest in this work.

\section{Funding}

The present study was supported by grants from the Foundation of Research project of health and Family Planning Commission of Jiading District, Shanghai (JDKW-2018-W15).

\section{Authors' Contributions}

$\mathrm{XZ}$ and PX had the study idea, wrote the proposal for the relevant committee, conducted the statistics and wrote the paper. WS and MX were responsible for data generation and participated in writing the paper, and conducted CT scans, helped prepare and analyze the data and proof-read the paper. XX participated in manuscript preparation. YY and LF were the study initiators, helped with the ethics proposal, data interpretation and proof-read the paper. All authors have read and approved the manuscript.

\section{Acknowledgements}

Not applicable.

\section{References}

1. Koval, K. J. et al. Ankle fractures in the elderly: what you get depends on where you live and who you see. Journal of Orthopaedic Trauma 19, 635-639 (2005).

2. Bartoníček, J. et al. Anatomy and classification of the posterior tibial fragment in ankle fractures. Archives of orthopaedic and traumatic surgery. Archiv fur orthopadische und Unfall-Chirurgie 135, 
505-516, doi:10.1007/s00402-015-2171-4 (2015).

3. Yasui, Y., Takao, M., Miyamoto, W., Innami, K. \& Matsushita, T. Anatomical reconstruction of the anterior inferior tibiofibular ligament for chronic disruption of the distal tibiofibular syndesmosis. Knee surgery, sports traumatology, arthroscopy: official journal of the ESSKA 19, 691-695, doi:10.1007/s00167-010-1311-1 (2011).

4. Haraguchi, N., Haruyama, H., Toga, H. \& Kato, F. Pathoanatomy of posterior malleolar fractures of the ankle. J Bone Joint Surg Am 88, 1085-1092, doi:10.2106/jbjs.E.00856 (2006).

5. Verhage, S. M., Krijnen, P., Schipper, I. B. \& Hoogendoorn, J. M. Persistent postoperative step-off of the posterior malleolus leads to higher incidence of post-traumatic osteoarthritis in trimalleolar fractures. Archives of orthopaedic and traumatic surgery. Archiv fur orthopadische und Unfall-Chirurgie 139, 323-329, doi:10.1007/s00402-018-3056-0 (2019).

6. Verhage, S. M., Schipper, I. B. \& Hoogendoorn, J. M. Long-term functional and radiographic outcomes in 243 operated ankle fractures. Journal of foot and ankle research 8, 45, doi:10.1186/s13047-0150098-1 (2015).

7. Xiaofeng G et al. Operative indications for posterior malleolar fractures. Chinese Journal of Orthopaedic Trauma 3 (2015).

8. Fu, S., Zou, Z. Y., Mei, G. \& Jin, D. Advances and disputes of posterior malleolus fracture. Chin Med J (Engl) 126, 3972-3977 (2013).

9. Siegler, S., Block, J. \& Schneck, C. D. The mechanical characteristics of the collateral ligaments of the human ankle joint. Foot \& ankle 8, 234-242, doi:10.1177/107110078800800502 (1988).

10. Chu, T. M., Reddy, N. P. \& Padovan, J. Three-dimensional finite element stress analysis of the polypropylene, ankle-foot orthosis: static analysis. Med Eng Phys 17, 372-379, doi:10.1016/13504533(95)97317-i (1995).

11. Anderson, D. D. et al. Physical validation of a patient-specific contact finite element model of the ankle. J Biomech 40, 1662-1669, doi:10.1016/j.jbiomech.2007.01.024 (2007).

12. Yu Z et al. Finite element modeling and mechanical analysis on supinationexternal rotation ankle injury. Journal of Medical Biomechanics 27, 282-288 (2012).

13. Shariff, S. S. \& Nathwani, D. K. Lauge-Hansen classification-a literature review. Injury 37, 888-890, doi:10.1016/j.injury.2006.05.013 (2006).

14. Ramos, L. S. et al. Evaluation of the Reproducibility of Lauge-Hansen, Danis-Weber, and AO Classifications for Ankle Fractures. Rev Bras Ortop (Sao Paulo) 56, 372-378, doi:10.1055/s-00401718508 (2021).

15. Yu, T., Zhang, Y., Zhou, H. \& Yang, Y. Distribution of posterior malleolus fracture lines in ankle fracture of supination-external rotation. Orthop Traumatol Surg Res 107, 103000, doi:10.1016/j.otsr.2021.103000 (2021).

16. De Vries, J. S., Wijgman, A. J., Sierevelt, I. N. \& Schaap, G. R. Long-term results of ankle fractures with a posterior malleolar fragment. The Journal of foot and ankle surgery: official publication of the American College of Foot and Ankle Surgeons 44, 211-217, doi:10.1053/j.jfas.2005.02.002 (2005). 
17. Mangnus, L. et al. Posterior Malleolar Fracture Patterns. J Orthop Trauma 29, 428-435, doi:10.1097/bot.0000000000000330 (2015).

18. Gardner, M. J., Brodsky, A., Briggs, S. M., Nielson, J. H. \& Lorich, D. G. Fixation of posterior malleolar fractures provides greater syndesmotic stability. Clin Orthop Relat Res 447, 165-171, doi:10.1097/01.blo.0000203489.21206.a9 (2006).

19. Miller, A. N., Carroll, E. A., Parker, R. J., Helfet, D. L. \& Lorich, D. G. Posterior malleolar stabilization of syndesmotic injuries is equivalent to screw fixation. Clin Orthop Relat Res 468, 1129-1135, doi:10.1007/s11999-009-1111-4 (2010).

20. Guangrong $\mathrm{Y}, \& \mathrm{Hao} \mathrm{H}$. Importance of the management of ankle fracture related problems. Chinese Journal of Anatomy and Clinics 24, 89-92 (2019).

21. Evers, J. et al. The role of a small posterior malleolar fragment in trimalleolar fractures: a biomechanical study. The bone \& joint journal 100-b, 95-100, doi:10.1302/0301-620x.100b1.Bjj2017-0435.R1 (2018).

\section{Figures}

\section{Figure 1}

Process of establishing a finite element analysis model of supination-external rotation ankle injury.

\section{Figure 2}

Boundary constraints and load diagram of damage model analysis 


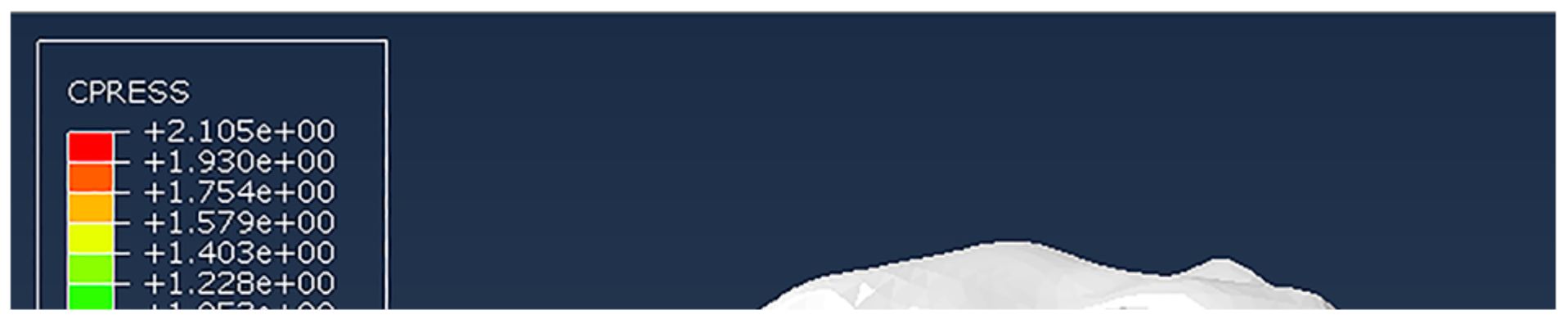

Figure 3

Validation of model validity by finite element analysis of supination-external rotation ankle injury.

\section{Figure 4}

Stress distribution during stage I supination-external rotation ankle injury.

\section{Figure 5}

Distribution of stress during injury to the anterior tibiofibular ligament in supination-external rotation ankle injury. 

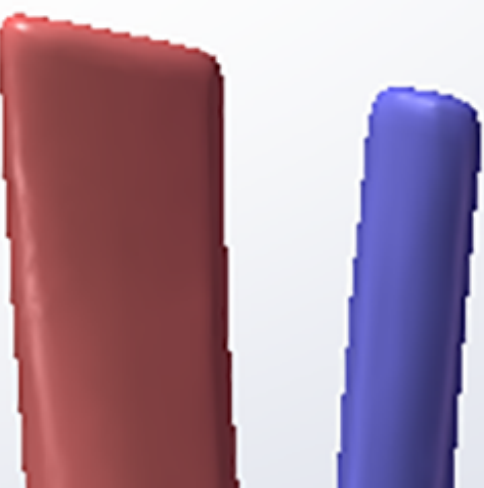

Figure 6

Model of lateral malleolar fracture in supination-external rotation ankle joint injury.

Figure 7 
Distribution of stress during injury of the lateral malleolar fracture in stage II supination-external rotation ankle injury.

\section{Figure 8}

Distribution of stress during injury of the posterior tibiofibular ligament with stage III supination-external rotation ankle injury.

\section{Figure 9}

Stress distribution during external ankle fracture associated with injury to the posterior tibiofibular ligament with stage III supination-external rotation ankle injury. 